Discrete Comput Geom 27:377-385 (2002)

DOI: $10.1007 / \mathrm{s} 00454-001-0075-2$

\title{
Separoids, Their Categories and a Hadwiger-Type Theorem for Transversals
}

\author{
J. L. Arocha, J. Bracho, L. Montejano, D. Oliveros, and R. Strausz \\ Instituto de Matemáticas, UNAM, \\ Circuito exterior, Ciudad Universitaria, \\ C.P. 04510, México DF, Mexico
}

\begin{abstract}
In this paper we study the topology of transversals to a family of convex sets as a subset of a Grassmanian manifold. This topology seems to be ruled by a combinatorial structure which we call a separoid. With these combinatorial objects and the topological notion of virtual transversal we prove a Borsuk-Ulam-type theorem which has as a corollary a generalization of Hadwiger's theorem.
\end{abstract}

\section{Introduction}

Suppose $\mathcal{F}$ is a family of convex sets in Euclidean $n$-space $R^{n}$. Then Helly's classical theorem asserts that if each $n+1$ members of $\mathcal{F}$ have a common point, there is a point common to all members of $\mathcal{F}$. It is natural to expect generalizations of this theorem replacing the concept of a common point ( 0 -transversal) by the concept of a $k$-plane that intersects all the convex sets ( $k$-transversal). The first attempts in this direction are due to Vincensini [19] and Klee [16], but, most significantly, to Hadwiger [13]. The next important contributions are due to Goodman and Pollack [11] who generalized Hadwiger's theorem from line transversals in $R^{2}$ to hyperplane transversals in $R^{n}$. There have been many more important contributions to the area of geometric transversal theory. For more on its history and literature see [6]-[8] and [12]. With this paper we emphasize that transversals, as a subset of a Grassmanian manifold, should be studied topologically (see also [3]), and that the topology of the space of transversals, as often appears in classic topology, seems to be ruled by simple combinatorial objects which we call Separoids. See also [4].

More precisely, if a family of convex sets in the plane has the property that every three of them have a transversal line, then the complete family does not necessarily have a transversal line. Adding the extra condition of a linear order such that every 
three sets are met by a line consistently with it, then Hadwiger proved that the complete family has a transversal line [13]. This result for higher dimensions is not true (see Fig. 1(b)). The generalizations of Hadwiger's theorem relevant here are due to Goodman and Pollack [11], to Pollack and Wenger [17] and to Anderson and Wenger [1]. All of them have as a hypothesis the existence of $k$-transversals to subfamilies consistent with a given combinatorial structure and as a conclusion the existence of a single transversal hyperplane.

Our generalization includes two new ideas. The first is to define a general combinatorial separation structure of the family of convex sets, on which a simple hypothesis is to be made. The second is to give as a conclusion what we call a virtual $k$-transversal, which is the existence of (homologically) as many transversal hyperplanes as if there was a $k$-transversal. This notion follows the spirit of Horn's theorem in [14].

In Section 2 we introduce and briefly study the notion of a separoid, which generalizes the previously used concepts of order type [10] and oriented matroid [2]. These combinatorial objects give information about the topology of the space of transversals. Namely, we prove a Borsuk-Ulam-type theorem for separoids which has as a corollary a new generalization of Hadwiger's theorem.

\section{The Categories of Separoids}

Definition 2.1. A separoid $S$ is a set together with a binary relation on its subsets, denoted $\mid$ and called the separation relation, that satisfies the following properties for $\alpha, \beta \subset S$ :

(i) $\alpha|\beta \Rightarrow \beta| \alpha$,

(ii) $\alpha \mid \beta \Rightarrow \alpha \cap \beta=\emptyset$,

(iii) $\alpha \mid \beta$ and $\alpha^{\prime} \subset \alpha \Rightarrow \alpha^{\prime} \mid \beta$.

When $\alpha \mid \beta$ we call it a separation of $S$, or say that " $\alpha$ is separated from $\beta$." If $S$ further satisfies that $\emptyset \mid S$, then it is called acyclic.

Example 1. Let $a_{0}, \ldots, a_{r}$ be points in some Euclidean (or affine) space. They define an acyclic separoid $S\left(a_{0}, \ldots, a_{r}\right)$ whose underlying set is $\left\{a_{0}, \ldots, a_{r}\right\}$, and two subsets $\alpha$ and $\beta$ are separated if there exists a hyperplane that leaves $\alpha$ on one side and $\beta$ on the other. That is,

$$
\alpha \mid \beta \Leftrightarrow\langle\alpha\rangle \cap\langle\beta\rangle=\emptyset,
$$

where \langle\rangle denotes the convex hull. These separoids are called point separoids.

Example 2. Let $\mathcal{F}=\left\{A_{0}, \ldots, A_{r}\right\}$ be a family of convex sets in some Euclidean space. It defines a separoid $S(\mathcal{F})$ with $\mathcal{F}$ as the underlying set and, again, with strict separation of subfamilies by hyperplanes as the separation relation. If the convex sets are compact, or bounded, then $S(\mathcal{F})$ is acyclic. We prove below that any acyclic separoid is isomorphic to one of these.

Observe that the separoid axioms are quite general because the sets $A_{i}$ need not be convex or even connected, however the separoid coincides with that of their convex hulls. 
Example 3. Given an oriented matroid, it naturally defines a separoid over the same base set by declaring that the negative part of each covector is separated from its positive part. The topes are then the maximal separations, so that the separoid has all the information of the oriented matroid. Hence separoids generalize oriented matroids. Observe that the oriented matroid is acyclic if and only if its separoid is acyclic.

Example 4. There are other natural separoids arising in various branches of mathematics. For example, for a topological space a separation relation arises by the existence of disjoint open neighborhoods.

Definition 2.2. Let $S$ and $T$ be separoids, and let $f: S \rightarrow T$ be a function:

- $f$ is a morphism if $\alpha \mid \beta$ in $T \Rightarrow f^{-1}(\alpha) \mid f^{-1}(\beta)$ in $S$,

- $f$ is a comorphism if $\alpha \mid \beta$ in $S \Rightarrow f(\alpha) \mid f(\beta)$ in $T$.

It is easy to see that separoids together with morphisms (or comorphisms) form a category. Both categories are important. We use the morphism category in this section, but in Section 4 comorphisms play an essential role. Furthermore, both types of functions arise in natural situations, as the following examples suggest.

Remark 1. Strong maps of oriented matroids yield morphisms of their separoids.

Remark 2. Let $\mathcal{F}=\left\{A_{0}, \ldots, A_{r}\right\}$ be a family of convex sets in $R^{n}$, and let $g: R^{n} \rightarrow$ $R^{m}$ be a linear (or affine) map. If $\mathcal{F}^{\prime}=\left\{g\left(A_{0}\right), \ldots, g\left(A_{r}\right)\right\}$, then the canonical bijection $S(\mathcal{F}) \rightarrow S\left(\mathcal{F}^{\prime}\right)$ is a morphism.

Remark 3. Let $\mathcal{F}$ be as above, and let $\mathcal{F}^{\prime}=\left\{B_{0}, \ldots, B_{r}\right\}$ be such that $B_{i} \subset A_{i}$, $i=0, \ldots, r$. Then the obvious correspondence $S(\mathcal{F}) \rightarrow S\left(\mathcal{F}^{\prime}\right)$ is a comorphism.

Observe that if $f: S \rightarrow T$ is a bijection, then it is a separoid morphism if and only if $f^{-1}$ is a comorphism. An isomorphism is a bijection which is both a morphism and a comorphism. An embedding is an injection which is a morphism and a comorphism; it is then an isomorphism with the separoid induced by its image (with the natural notion of "induced by").

Theorem 2.1 (Realization). Let $S$ be a finite acyclic separoid. Then there exists a family of convex sets $\mathcal{F}$ in some Euclidean space such that $S$ is isomorphic to $S(\mathcal{F})$.

Proof. ${ }^{1} \quad$ The basic idea is to embed $S$ canonically in a separoid of bounded convex sets. For this, let $\Delta$ denote the acyclic separoid over the set $\{-, 0,+\}$ with the unique nontrivial separation $\{-\} \mid\{+\}$ (where trivial separations are those of the empty set).

${ }^{1}$ We thank E. Schepin for his collaboration in this proof. 
Then, for every separation $\alpha \mid \beta$ in an acyclic separoid $S$, we have a characteristic morphism

$$
\begin{gathered}
\chi_{\alpha \mid \beta}: S \rightarrow \Delta, \\
\chi_{\alpha \mid \beta}(x)= \begin{cases}- & \text { if } x \in \alpha, \\
+ & \text { if } x \in \beta, \\
0 & \text { otherwise. }\end{cases}
\end{gathered}
$$

Now, in the category of separoids and morphisms, the categorical product is defined as follows. Let $S$ and $T$ be separoids; their product $S \times T$ has base set $S \times T$ and separations

$$
\alpha\left|\beta \Longleftrightarrow \pi_{S}(\alpha)\right| \pi_{S}(\beta) \text { or } \pi_{T}(\alpha) \mid \pi_{T}(\beta),
$$

where $\pi_{S}$ and $\pi_{T}$ are the projections. It is easy to see that for an acyclic separoid $S$,

$$
\begin{gathered}
\chi: S \rightarrow \prod_{\alpha \mid \beta} \Delta, \\
\chi:=\prod_{\alpha \mid \beta} \chi_{\alpha \mid \beta}
\end{gathered}
$$

is an embedding, where the product is taken over all separations of $S$. We can therefore conclude that any finite separoid is embedded in a finite product of copies of $\Delta$. Thus, we are left to prove that $\Delta^{n}:=\prod^{n} \Delta$ is isomorphic to a separoid of convex sets.

To see this let $\mathcal{B}=\{[-1,0),[-1,1],(0,1]\}$. It is a family of convex sets in $\mathbb{R}$, where the separations are given by points, thus $\Delta \cong S(\mathcal{B})$. Given two families of convex sets $\mathcal{F}$ and $\mathcal{G}$, define $\mathcal{F} \times \mathcal{G}=\{A \times B \mid A \in \mathcal{F}, B \in \mathcal{G}\}$, a family of convex sets in the product of the ambient spaces. It is also easy to prove that

$$
\Delta^{n} \cong S\left(\mathcal{B}^{n}\right):=S\left(\prod^{n} \mathcal{B}\right)
$$

and we are done.

The theorem can be refined to the realization by families of compact convex sets by taking $\mathcal{B}_{\varepsilon}=\{[-1,-\varepsilon],[-1,1],[\varepsilon, 1]\}$ with $0<\varepsilon \leq 1$; and proving that $\Delta^{n} \cong S\left(\mathcal{B}_{\varepsilon}^{n}\right)$ if and only if $0<\varepsilon \leq 1 /(2 n-1)$.

This concludes the proof of the theorem. The reader may recognize in it a classic idea of Alexsandroff.

From now on we assume that separoids are finite and acyclic. The realization theorem allows us to define the geometric dimension of separoids (see also [5]).

Definition 2.3. Given a separoid $S$, its geometric dimension, $\operatorname{dim}_{g}(S)$, is the least $k$ for which there exists a family $\mathcal{F}$ of convex sets in $\mathbb{R}^{k}$ such that $S \cong S(\mathcal{F})$.

A straightforward generalization of Kirchberger's theorem [15] (see (2.4) of [6]) to families of convex sets gives the following characterization of separations by small subfamilies. 
Theorem 2.2 (Kirchberger). Let $S$ be a separoid with $\operatorname{dim}_{g}(S) \leq k$. Then $\alpha \mid \beta$ if and only if for every $S^{\prime} \subset S$ with $\# S^{\prime} \leq k+2$, we have that $\left(S^{\prime} \cap \alpha\right) \mid\left(S^{\prime} \cap \beta\right)$.

This yields a criterion for a function to be a comorphism which is used in the last section.

Corollary 2.1. Let $S$ and $T$ be separoids and let $f: S \rightarrow T$ be a function. If $\operatorname{dim}_{g}(T) \leq$ $k$, then $f$ is a comorphism if and only if for every $S^{\prime} \subset S$ with $\# S^{\prime} \leq k+2$, we have that $\left.f\right|_{S^{\prime}}$ is a comorphism.

Proof. One direction is obvious. Now, suppose $\alpha \mid \beta$ in $S$; we must prove that $f(\alpha) \mid$ $f(\beta)$ in $T$. For this we use the criterion of Theorem 2.2. Let $T^{\prime} \subset T$ be such that $\# T^{\prime} \leq k+2$. We can easily construct $S^{\prime} \subset S$ such that $\# S^{\prime} \leq k+2, f\left(S^{\prime} \cap \alpha\right)=T^{\prime} \cap f(\alpha)$ and $f\left(S^{\prime} \cap \beta\right)=T^{\prime} \cap f(\beta)$. Since $\left.f\right|_{S^{\prime}}$ is a comorphism and $S^{\prime} \cap \alpha \mid S^{\prime} \cap \beta$ in $S$, then $T^{\prime} \cap f(\alpha) \mid T^{\prime} \cap f(\beta)$ in $T$.

\section{Virtual Transversals}

If three plane convex sets intersect pairwise, they do not necessarily have a common point, nevertheless they do have a common transversal line. However, there is not only one such transversal line. In fact, there are as many, from the homological point of view, as there are lines through a point - that is, we can have a line moving in the plane, always transversal to the convex sets, and coming back to itself with the opposite orientation, see Fig. 1(a). In this sense, we may think that we have a "virtual" point transversal to our convex sets. As a particular case of Corollary $4.2(k=0)$, this will be proved for any family of more than three convex sets.

As stated in the Introduction, Hadwiger [13] proved that if every three sets of a family of plane convex sets are met by a line consistently with some given order, then the complete family has a transversal line. This result is not true for convex sets in Euclidean three space. For a simple example consider the four convex sets shown in Fig. 1(b). It is easy to see that there is no transversal line to these convex sets, but every three of them are met by a line consistently with the ordering. However, there are many transversal planes to the four convex sets (because convex set 1 "sees" Fig. 1(a)). In fact, there are as many transversal planes, from the homological point of view, as there are planes through

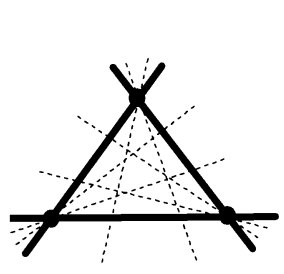

(a)

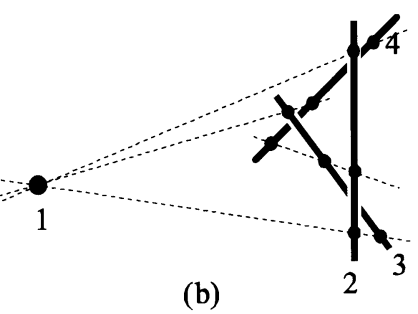

(b)

Fig. 1 
a line, and in this sense we may think that there is a "virtual" transversal line to them. This motivates our next definitions.

All our homology and cohomology groups are with $Z_{2}$ coefficients. By $G_{n}$ we denote the space of hyperplanes in $\mathbb{R}^{n+1}$. It is retractable to the classic Grassmannian of codimension 1 subspaces, and therefore homotopy equivalent to $\mathbb{P}^{n}$.

Definition 3.1. For a family $\mathcal{F}$ of convex sets, we denote by $\mathcal{T}(\mathcal{F})$ the subspace of $G_{n}$ consisting of all hyperplanes transversal to $\mathcal{F}$.

Definition 3.2. Let $\mathcal{F}=\left\{A_{0}, \ldots, A_{r}\right\}$ be a family of convex sets in $\mathbb{R}^{n+1}$. We say that $\mathcal{F}$ has a virtual $k$-transversal if the homomorphism induced by the inclusion

$$
H_{n-k}(\mathcal{T}(\mathcal{F})) \rightarrow H_{n-k}\left(G_{n}\right)
$$

is nonzero.

In particular, if $L$ is a $k$-transversal to $\mathcal{F}$, then $\mathcal{F}$ has a virtual $k$-transversal, because if $\hat{L}$ is the set of all hyperplanes through $L$, then $H_{n-k}(\hat{L}) \rightarrow H_{n-k}\left(G_{n}\right)$ is not zero (in fact, $H_{n-k}\left(G_{n}\right)$ is generated by the fundamental class of $\left.\hat{L} \subset G_{n}\right)$, and since $\hat{L} \subset \mathcal{T}(\mathcal{F})$, then $H_{n-k}(\mathcal{T}(\mathcal{F})) \rightarrow H_{n-k}\left(G_{n}\right)$ is nonzero.

Our next theorem states that for a family of $k+2$ convex sets, the notions of virtual $k$ transversal and $k$-transversal coincide. The proof is simple thanks to a classic technique in fiber bundles.

Theorem 3.1. Let $\mathcal{F}=\left\{A_{0}, \ldots, A_{k+1}\right\}$ be a family of convex sets in $\mathbb{R}^{n+1}$, with $k \leq n$. Then $\mathcal{F}$ has a virtual $k$-transversal if and only if it has a $k$-transversal.

Proof. We have just proved one implication. For the other, consider $\hat{\mathcal{T}}(\mathcal{F})=\left\{\left(H, a_{0}\right.\right.$, $\left.\left.\ldots, a_{k+1}\right) \mid H \in \mathcal{T}(\mathcal{F}), a_{i} \in H \cap A_{i}\right\}$ with the two natural projections giving the diagram

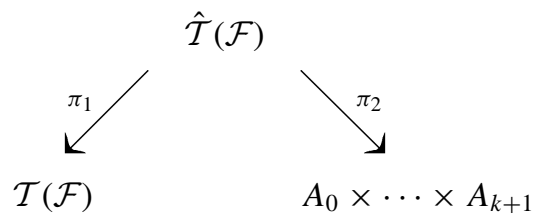

Observe that $\pi_{1}$ is a homotopy equivalence because the fiber $\pi_{1}^{-1}(H)=\prod_{i=0}^{k+1}\left(H \cap A_{i}\right)$ is contractible for every $H \in \mathcal{T}(\mathcal{F})$. On the other hand, suppose there is no $k$-transversal to $\mathcal{F}$. Then each collection of points $\left(a_{0}, \ldots, a_{k+1}\right)$ with $a_{i} \in A_{i}$ determines a unique $(k+1)$-plane $L$ in $\mathbb{R}^{n+1}$. Then $\pi_{2}^{-1}\left(a_{0}, \ldots, a_{k+1}\right)$ consists of the hyperplanes that contain $L$, which is homeomorphic to $\mathbb{P}^{2-k-1}$. Furthermore, it is easy to see that $\pi_{2}$ is a fiber bundle with fiber $\mathbb{P}^{n-k-1}$. Since its base is contractible, then $\hat{\mathcal{T}}(\mathcal{F})$, and hence $\mathcal{T}(\mathcal{F})$, has the homotopy type of $\mathbb{P}^{n-k-1}$. Finally, this implies that the homeomorphism $H_{n-k}(\mathcal{T}(\mathcal{F})) \rightarrow$ $H_{n-k}\left(G_{n}\right)$ is zero, and therefore $\mathcal{F}$ does not have a virtual $k$-transversal. 


\section{Borsuk-Ulam for Separoids}

The purpose of this section is to establish the following Borsuk-Ulam-type theorem for separoids which has as a corollary a generalization of Hadwiger's theorem for transversals. Its proof follows the ideas of Pollack and Wenger in [17]. Furthermore, this theorem is related with the results of Goodman and Pollack in [11] and Anderson and Wenger in [1]. All these previous results have as a conclusion the existence of one transversal hyperplane, but our results follow the spirit of Horn's theorem [14] in which the conclusion states the existence of a virtual $k$-transversal, that is, of homologically as many transversal hyperplanes as the hyperplanes that contain a fixed $k$-plane.

Theorem 4.1. Let $\mathcal{F}=\left\{A_{0}, \ldots, A_{r}\right\}$ be a family of convex sets in $\mathbb{R}^{n+1}$. If there exists a separoid comorphism

$$
\varphi: S(\mathcal{F}) \rightarrow T
$$

where $\operatorname{dim}_{g}(T) \leq k \leq n$, then $\mathcal{F}$ has a virtual $k$-transversal.

Proof. By Remark 3, we may assume that $T$ is a separoid of points in $\mathbb{R}^{k}$. Suppose that $\mathcal{F}$ does not have a virtual $k$-transversal, that is, that the homomorphism induced by the inclusion

$$
H_{n-k}(\mathcal{T}(\mathcal{F})) \rightarrow H_{n-k}\left(G_{n}\right)
$$

is zero.

Let $U=\left\{v \in S^{n} \mid\right.$ there is no $H \in \mathcal{T}(\mathcal{F})$ such that $\left.H \perp v\right\}$. Note that $U=-U$. Let $U^{\prime} \subset \mathbb{P}^{n}$ be the projection of $U \subset S^{n}$ in $\mathbb{P}^{n}$. Observe that the canonical retraction that takes $G_{n}$ to $\mathbb{P}^{n}$, takes $\mathcal{T}(\mathcal{F})$ to $\mathbb{P}^{n}-U^{\prime}$, so that (1) implies that the homomorphism induced by the inclusion

$$
H_{n-k}\left(\mathbb{P}^{n}-U^{\prime}\right) \rightarrow H_{n-k}\left(\mathbb{P}^{n}\right)
$$

is zero. By Alexander Duality, this implies that, in cohomology, the homomorphism induced by the inclusion

$$
H^{k}\left(\mathbb{P}^{n}\right) \rightarrow H^{k}\left(U^{\prime}\right)
$$

is nonzero.

If $v \in U$, then the hyperplanes perpendicular to $v$ that yield a nontrivial separation of $\mathcal{F}$ form an open interval; let $H_{v}$ be the one at the middle. Clearly, $H_{v}=H_{-v}$. Let $H_{v}^{+}$ and $H_{v}^{-}$be the two open half-spaces determined by $H_{v}$, so that $H_{v}^{+}=H_{-v}^{-}$.

Observe that

$$
p(v)=\sum_{i=0}^{r} d\left(A_{i}, H_{v}^{+}\right)
$$

where $d\left(A_{i}, H_{v}^{+}\right)$denotes the infimum of the distances of points in $A_{i}$ and points in $H_{v}^{+}$, is never zero and that it depends continuously on $v \in U$. Therefore, we have a 
continuous map

$$
\begin{array}{r}
f: U \rightarrow \mathbf{R}^{k}, \\
f(v)=\sum_{i=0}^{r} \frac{d\left(A_{i}, H_{v}^{+}\right)}{p(v)} \varphi\left(A_{i}\right) .
\end{array}
$$

Recall that $U=-U$ is an open set of $S^{n}$ and that (2) is nonzero. By the Borsuk-Ulam theorem [18], there exists $v_{0} \in U$ for which $f\left(v_{0}\right)=f\left(-v_{0}\right)$.

Let $\alpha=\left\{A \in \mathcal{F} \mid A \subset H_{v_{0}}^{+}\right\}$and $\beta=\left\{A \in \mathcal{F} \mid A \subset H_{-v_{0}}^{+}\right\}$. By definition, $\alpha$ is separated from $\beta$ and hence, since $\varphi$ is a comorphism, $\varphi(\alpha)$ is separated from $\varphi(\beta)$. On the other hand, note that $f\left(v_{0}\right)$ is a convex combination of the points $\varphi(\beta)$ and also $f\left(-v_{0}\right)$ is a convex combination of the points $\varphi(\alpha)$. Hence, $f\left(v_{0}\right)=f\left(-v_{0}\right) \in$ $\langle\varphi(\alpha)\rangle \cap\langle\varphi(\beta)\rangle \neq \emptyset$, which is a contradiction.

Corollary 4.1. Let $\mathcal{F}$ be a family of convex sets in $\mathbb{R}^{n+1}$, and let $T$ be a separoid with $\operatorname{dim}_{g}(T) \leq k$. Suppose there exists a function $f: \mathcal{F} \rightarrow T$ such that for every subfamily $\mathcal{F}^{\prime} \subset \mathcal{F}$ with $\# \mathcal{F}^{\prime}=k+2$, the restriction $f: S\left(\mathcal{F}^{\prime}\right) \rightarrow T$ is a comorphism, then $\mathcal{F}$ has a virtual $k$-transversal.

Proof. Follows immediately from Corollary 2.1 and Theorem 4.1.

Definition 4.1. Let $\mathcal{F}=\left\{A_{0}, \ldots, A_{r}\right\}$ be a family of convex sets in $\mathbb{R}^{n+1}$, and let $B=\left\{b_{0}, \ldots, b_{r}\right\}$ be a family of points in $\mathbb{R}^{k}, k \leq n$. A $k$-plane $L$ transversal to $\mathcal{F}^{\prime}=\left\{A_{i_{0}}, A_{i_{1}}, \ldots, A_{i_{k+1}}\right\}$ is consistent with $B$ if there are points $a_{i_{j}} \in A_{i_{j}} \cap L$ such that the correspondence $\left(a_{i_{j}} \rightarrow b_{i_{j}}\right)$ is an isomorphism from the separoid $S\left(a_{i_{0}}, \ldots, a_{i_{k+1}}\right)$ to the separoid $S\left(b_{i_{0}}, \ldots, b_{i_{k+1}}\right)$.

Corollary 4.2 (Hadwiger-Type Theorem). Let $\mathcal{F}=\left\{A_{0}, \ldots, A_{r}\right\}$ be a family of convex sets in $\mathbb{R}^{n+1}$, and let $B=\left\{b_{0}, \ldots, b_{r}\right\}$ be a family of points in $\mathbb{R}^{k}, k \leq n$. If for every subfamily $\mathcal{F}^{\prime} \subset \mathcal{F}$ with $\# \mathcal{F}^{\prime}=k+2$ there exists a $k$-transversal to $\mathcal{F}^{\prime}$ consistent with $B$, then $\mathcal{F}$ has a virtual $k$-transversal.

Proof. For every subfamily $\mathcal{F}^{\prime} \subset \mathcal{F}$ with $\# \mathcal{F}^{\prime}=k+2$ there exists a $k$-plane $L$ transversal to $\mathcal{F}^{\prime}=\left\{A_{i_{0}}, A_{i_{1}}, \ldots, A_{i_{k+1}}\right\}$ with the property that there are points $a_{i_{j}} \in A_{i_{j}} \cap L$ such that the correspondence $\left(a_{i_{j}} \rightarrow b_{i_{j}}\right)$ is an isomorphism from the separoid $S\left(a_{i_{0}}, \ldots, a_{i_{k+1}}\right)$ to the separoid $S\left(b_{i_{0}}, \ldots, b_{i_{k+1}}\right)$. Thus, by Corollary $2.1, S\left(a_{i_{0}}, \ldots, a_{i_{k+1}}\right) \rightarrow S(B)$ is a comorphism. However, by Remark $3, S\left(\mathcal{F}^{\prime}\right) \rightarrow S\left(a_{i_{0}}, \ldots, a_{i_{k+1}}\right)$ is also a comorphism. So, this corollary is implied by the preceding one, since the composition of comorphisms is a comorphism.

Corollary 4.2 is related but does not generalize the results of Anderson and Wenger in [1] and of Goodman and Pollack in [11]. The hypothesis in these theorems is that there exists a $k$-transversal to each subfamily of size $k+2$ consistent with a given oriented matroid. The hypothesis in Corollary 4.2 assumes that there exists a $k$-transversal to each subfamily of size $k+2$ consistent with a given realizable oriented matroid, that 
is, an oriented matroid which can be represented by a set of points in $R^{k}$. Realizability is required in order to create the separoid comorphism needed to apply Theorem 4.1. Observe however that our conclusion is stronger. We conjecture that under the hypothesis in [1] and [11] it is also possible to conclude the existence of a virtual $k$-transversal.

\section{References}

1. L. Anderson and R. Wenger, Oriented matroids and hyperplane transversals, Adv. in Math. 119 (1996), $117-125$.

2. A. Björner, M. Las Vergnas, B. Sturmfels, N. White and G. Ziegler, Oriented Matroids, Encyclopedia of Mathematics, Cambridge University Press, Cambridge, 1993.

3. J. Bracho and L. Montejano, Helly type theorems on the homology of the space of transversals. Discrete Comput. Geom., this issue, pp. 387-393.

4. J. Bracho, L. Montejano and D. Oliveros, The topology of the space of transversals through the space of configurations, Topology Appl., to appear.

5. J. Bracho and R. Strausz, Separoids and a characterization of linear acyclic uniform oriented matroids, Preprint, 2001.

6. L. Danzer, B. Grünbaum and V. Klee, Helly's theorem and its relatives, in Convexity, Proceedings of Symposia in Pure Mathematics, vol. 7, American Mathematical Society, Providence, RI, 1963, pp. 101180.

7. J. Eckhoff, Helly, Radon, and Carathéodory type theorems, in Handbook of Convex Geometry (P. M. Gruber and J. M. Wills, eds.), Elsevier, Amsterdam, 1993, pp. 389-447.

8. P. Erdôs and G. Purdy, Extremal problems in combinatorial geometry, in Handbook in Combinatorics (R. L. Grahamm, M. Grötschel and L. Lovász, eds.), North-Holland, Amsterdam, 1995, pp. 809-874.

9. J. E. Goodman, When is a set of lines in space convex?, Notices Amer. Math. Soc. 45 (1998), 222-232.

10. J. E. Goodman and R. Pollack, Multidimentional sorting, SIAM J. Comput. 12 (1983), 484-503.

11. J. E. Goodman and R. Pollack, Hadwiger's transversal theorem in higher dimensions, J. Amer. Math. Soc. 1 (1988), 301-309.

12. J. E. Goodman, R. Pollack and R. Wenger, Geometric transversal theory, in New Trends in Discrete and Combinatorial Geometry (J. Pach, ed.), Springer-Verlag, Berlin, 1993, pp. 163-198.

13. H. Hadwiger, Über Eibereiche mit gemeinsamer Treffgeraden, Portugal Math. 16 (1957), 23-29.

14. A. Horn, Some generalizations of Helly's theorem on convex sets. Bull. Amer. Math. Soc. 55 (1949), 923-929

15. P. Kirchberger, Über Tschebyschefche Annäherungsmethoden, Math. Ann. 57 (1903), 509-540.

16. V. Klee, Common secants for plane convex sets, Proc. Amer. Math. Soc. 5 (1954), 639-641.

17. R. Pollack and R. Wenger, Necessary and sufficient conditions for hyperplane transversals, Combinatorica 10 (1990), 307-311.

18. E. H. Spanier, Algebraic Topology, McGraw-Hill, New York, 1966.

19. P. Vincensini, Figures convexes et variétés linéaires de l'espace euclidien à $n$ dimensions, Bull. Sci. Math. 59 (1935), 163-174.

Received October 25, 2000, and in revised form September 27, 2001, October 6, 2001, and October 11, 2001.

Online publication March 1, 2002. 